\title{
Silos musulmanes en la llamada "Casa de San Isidro» (Madrid)
}

\author{
Miguel Angel lopez Marcos * \\ Elena Serrano Herrero \\ Olga Vallespin
}

El solar conocido más comúnmente como "Casa de San Isidro" se encuentra situado en la Costanilla de San Pedro, número 12-14 (Madrid) y ha sido objeto de una excavación sistemática durante los meses de mayo a octubre de 1989. Excavaciones de este tipo ya se vienen realizando desde varios años atrás en todo solar que posea cierto valor arqueológico, sin embargo nos hallamos en uno de los casos de mayor relevancia desde varios puntos de vista. Por una parte el solar constituye la base de una de las iniciativas más importantes de cara a la capitalidad cultural de Europa en 1992. La Comunidad de Madrid promovió entonces la excavación total del recinto que, si bien inconclusa por ahora, permite ya la elaboración de una serie de hipótesis de trabajo, a la vez que la comprobación o corrección de otros datos de los que se tenía constancia por escrito; éstos nos fueron facilitados por $F$. Marín Parellón y el resto del "Equipo Madrid». Parte de los restos hallados serán incluidos como zonas visitables en la construcción del museo de Madrid Medieval y $S$. Isidro a partir de un proyecto elaborado por V. Quintas Ripoll.

Por otra parte hemos de aducir una importancia puramente histórica y arqueológica, por hallarse ubicado en una zona que fue con toda probabilidad arrabal musulmán; y con posterioridad quedó englobado en el interior del segundo recinto amurallado en un lugar muy próximo a la Puerta de Moros.

Departamento de Prehistoria e Historia Antigua de la UNED. 
En cuanto a los resultados de la excavación propiamente dichos, podemos resaltar la existencia de tres núcleos fundamentales; en primer lugar la localización de la práctica totalidad de los cimientos del palacio de los Condes de Paredes construido en el siglo XVI; la planta del mismo queda reflejada en la figura 1 , y se ajusta en gran medida a los datos aportados por el Equipo Madrid. Los límites actuales del solar coinciden con los del palacio propiamente dicho, entendiendo que la zona norte no seria residencia habitual, sino que estaría reservada para cocheras y caballerizas. De forma de trapecio irregular, dispone la zona habitacional de forma perimétrica en torno a un patio rectangular centralizado limitado por unas columnas de claro estilo renacentista perteneciente a la escuela formada en Madrid con la construcción del Alcazar. En el ángulo SO del patio y ya en la zona porticada queda ubicado el pozo donde según la tradición S. Isidro realizó el milagro de todos conocido. En la actualidad aun conserva agua $(3 \mathrm{~m})$ con una profundidad total de $21 \mathrm{~m}$.

Otro de los núcleos a destacar corresponde al cementerio de San Andrés de cuya existencia ya se tenía constancia; en las fuentes históricas no se precisan con detalles los limites, si bien, quedaría englobado entre el jardín de la parroquia de San Andrés y el sector Oeste del solar. Ha sido localizado parte de él entre el ábside de la Capilla del Obispo y la esquina NO del palacio (fig. 1). Fueron localizados los restos de hasta nueve individuos, la mayoria de ellos fragmentados y en un estrato revuelto con excepción de los individuos, un niño y un adulto que conservan su deposición original (decubito supino). La mayoria de ellos muestran una orientación Oeste-Este.

La potencia estratigráfica de todo el conjunto ha sido más bien escasa. Esto ha limitado las posibilidades, como es lógico, a la hora de establecer conclusiones debiendo optar en todo momento por otros tipos de fundamentos. Dicho problema no es exclusivo del solar; por contra, en otros puntos como Plaza de los Carros, Angosta de los Mancebos o Cava Baja, por escoger los más cercanos, se ha planteado el mismo problema por hallarse todos en las inmediaciones del antiguo cerro de $\mathrm{S}$. Andrés y ser rebajado de forma continuada a lo largo de toda la historia urbanistica de la zona.

En el caso que nos ocupa, toda se agrava al sufrir en 1974 la demolición y arrasamiento definitiva del palacio que con toda seguridad destruyó la mayoría de los escasos niveles existentes. Ante estas perspectivas, la potencia estratigráfica se queda reducida principalmente a un nivel superficial revuelto como producto de la demolición de unos 30-60 $\mathrm{cm}$ de espesor; en gran parte del solar se localiza inmediatamente después el terreno base natural arcilloso compuesto de "arena de miga". 


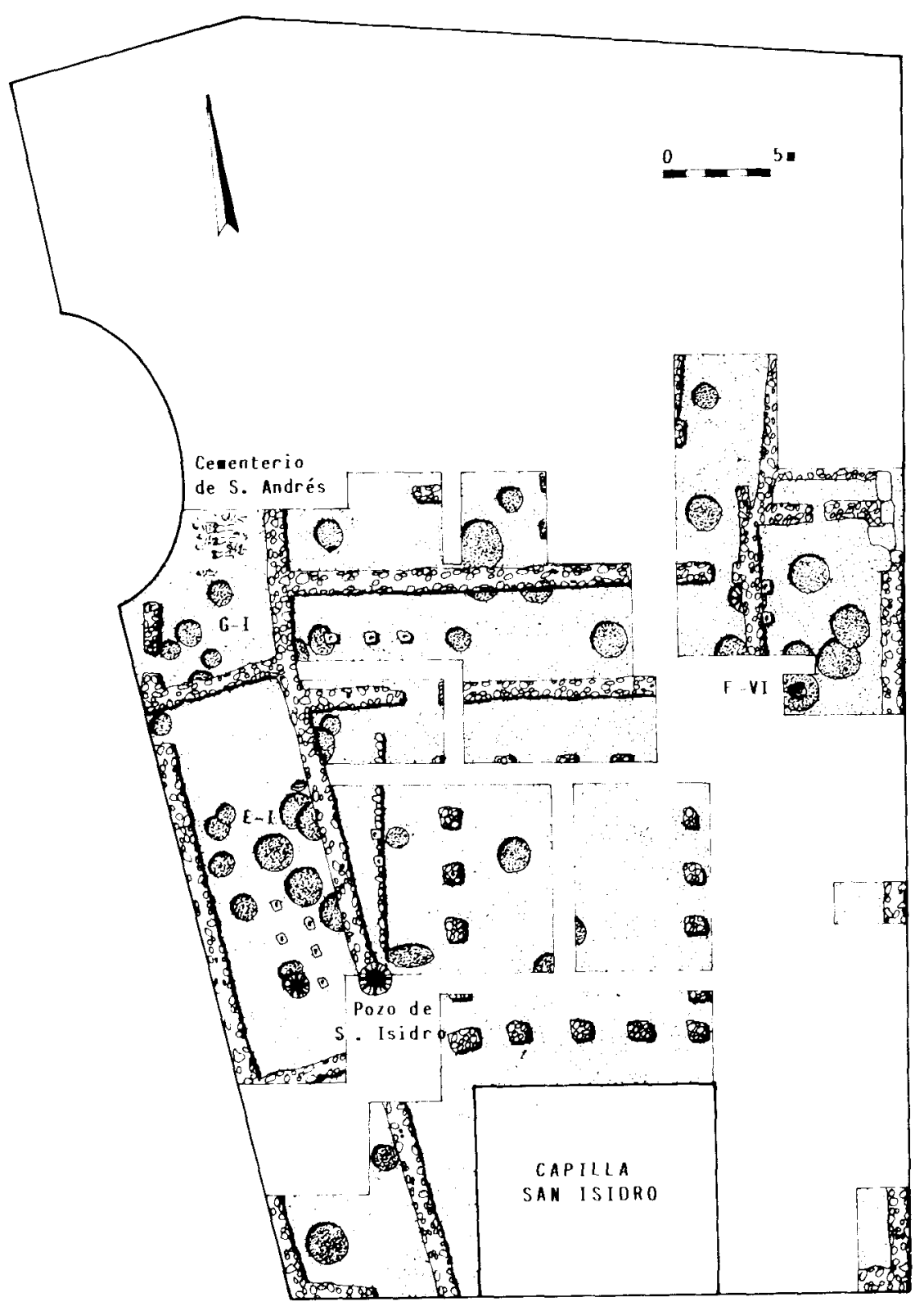

Fig. 1. Planimetria del solar. La zona punteada indica el área excavada. Planta del palacio de los Condes de Paredes, dispersión de silos y pozos y restos del comenterio de S. Andrés. Dibujo de Miguel Angel López Marcos. 
Únicamente en parte del patio podemos encontrar un nivel intermedio, asi como en otras estructuras de tipo subterráneas que por ser conjuntos cerrados de mayor profundidad no se han visto afectados por las remodelaciones de la época moderna. Nos encontramos pues, con pozos y silos que constituyen el tercer núcleo a destacar en el solar. En otras zonas próximas como la plaza de los Carros o la Cava Baja también han sido documentadas estructuras de este tipo u otras como cuevas o qanat también en la Plaza de los Carros y siempre de tipo subterráneo.

Lo más revelador de los silos y pozos de la "Casa de San Isidro", no radica pues en su aspecto innovador para el conocimiento de un arrabal musulmán; de éste y de los silos de la zona ya se tenía constancia con anterioridad. De los aproximadamente 2000 metros cuadrados que posee el solar, han sido excavados dos tercios con óptimos resultados al iocalizar 45 silos y pozos, de los cuales se han excavado diez. En una próxima campaña será excavado el resto, con lo cual se podrá disponer de una visión de conjunto mucho mayor que en otros solares donde las excavaciones practicadas tenian un carácter de urgencia mucho más acusado reduciéndose a pequeños sondeos o catas. Aqui por vez primera se puede contemplar la dispersión de silos y pozos en una extensión excavada que podemos considerar como representativa. La disposición anárquica de todos ellos junto con la forma y tamaño de los mismos (todos diferentes) obedece a una implantación diacrónica y que sólo respondia a necesidades del momento.

Se extienden por todo el solar pero hay dos pequeños grupos, al Este y otro al Oeste, en torno al E-I y al F-VI respectivamente que concentran casi la mitad del total. Todos ellos presentan una sección de la boca circular u ovoide; su contorno y tamaño varia enormemente como ya hemos comentado, aunque responden a una forma típica de "fondo de saco" buscando siempre la máxima capacidad de relación a la mayor resistencia de las paredes (fig. 2.2).

Lógico es, pensar que no fueron construidos a la vez, pero es difícil de precisar el periodo que abarca su dispersión y desarrollo. De un lado existe el conjunto ergológico de algunos de ellos que hasta el momento, no es esclarecedor ya que se muestra, desde el punto de vista cerámico muy homogéneo relacionándose con la cerámica de otros yacimientos de la Marca Media fechado en torno a los siglos $x$ y $x$. Por otra parte, dicho material no tiene por qué corresponderse cronológicamente con el periodo en que fueron construidos y utilizados los silos. Igualmente, al ser conjuntos cerrados sin un apoyo estratigráfico ni ocupacional, se dificulta su relación entre ellos mismos, salvo en dos casos de entrecruzamiento donde se ha podido poner de manifiesto dicha relación. 

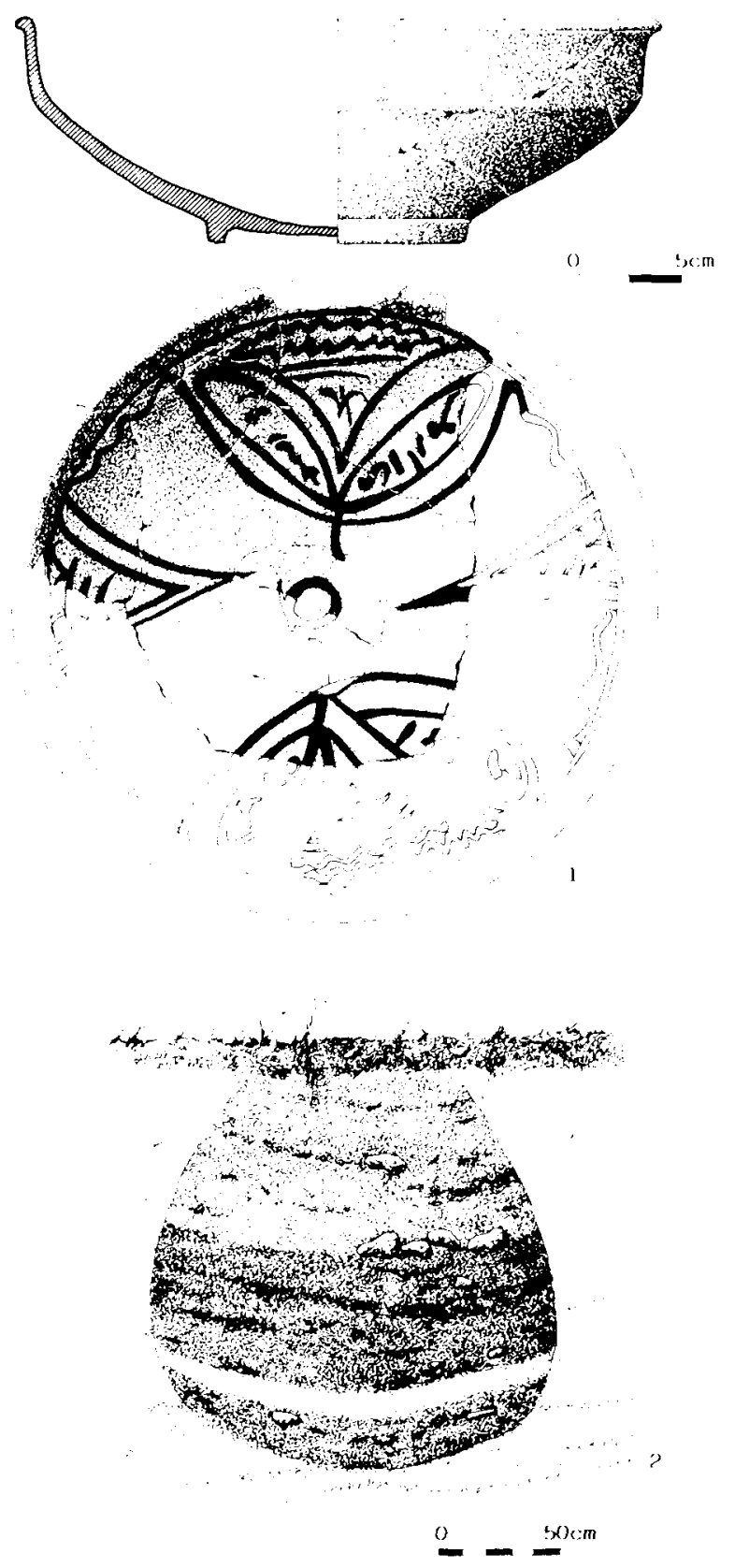

Fig. 2.1. Ataifor con decoración vegetal de manganeso sobre vedrío melado; 2 , Sección del silo A-I. Dibujo de Miguel Angel López Marcos. 
En el E-I existe uno de ellos (fig. 1); aqui se documentaron dos silos donde se pudo comprobar que poseían un contenido de materiales muy similar a la vez que las tierras aparecidas en dos distintos niveles presentaban unas características muy similares en cuanto a calidad y textura. Cabe suponer pues, que la construcción de uno y otro silo están separados por un corto espacio de tiempo, si no responde a un simple ensanchamiento por necesidades del momento.

En el F-VI (fig. 1) se nos plantea un caso similar, pero que obedece a otro tipo de planteamiento. Tras excavar el situado más al Norte se pudo comprobar que en la zona SO del hueco quedaba plasmada la huella de parte del otro silo que había sido recortado al construir aquel. Color textura y grado de compactación de uno y otro eran acusadamente distintos. Queda claro pues que, una vez construido en origen el situado más al SO, pasó un tiempo de utilización más o menos amplio, otro período destinado a la reutilización como basurero hasta su colmatación, para posteriormente pasar un período de tiempo determinado hasta que se construyera el otro más al Norte, de forma que el grado de compactación alcanzado por aquel, actuara como parte de la pared del nuevo silo, sin que ésta se viniera abajo. El próximo estudio de los materiales de unos y otros permitirá sin duda la elaboración de nuevas conclusiones que se aproximen a alguna fijación cronológica.

El contenido de los silos y pozos excavados es muy homogéneo a nivel general. La abundancia de desechos de materiales de construcción dan una muestra clara de que efectivamente estarian asociados a niveles de habitación hoy perdidos (ladrillos, tejas, piedras, etc.). Se completa dicho contenido con cenizas, carbón, huesos, metales, cerámicas, etc. Desde el punto de vista ceramológico nos encontramos con un muestrario homogéneo típicamente musulmán a excepción de uno de los pozos donde se entremezcla con la llamada cerámica de "repoblación» (fig. 5); tipológicamente son muy similares a las aparecidas en Alcalá la Vieja, fechadas en torno a los siglos XII-XIII. El muestrario es bastante complejo, con un amplio repertorio de cerámica no vidriada (fig. 4) y vidriada (fig. 2.1). Paralelos encontramos a lo largo de toda la Marca Media (Qal'at Abd-al-Salam, Talamanca del Jarama, Toledo, Guadalajara, Vascos, Cervera, Olmos, Calatalifa, Melque, etc. En cuanto a las formas más comunes halladas podemos destacar: jarras, jarritas, vasos, tarros, arcaduces, fuentes, ataifores, platos, cuencos, escudillas, cazuelas, ollas, tazas, candiles, cántaros, tapaderas, tinajas, anafres, lebrillos, orzas, redomas, etc.

Desde un punto de vista puramente decorativo podemos reseñar que los motivos más abundantes en las cerámicas no vidriadas son sin duda alguna los pintados conocidos comúnmente como "dedos de fátima" en 
rojo o negro (fig. 4.2), también abundan las pintadas al interior o/y al exterior generalmente en rojo ya sea parcial o total (fig. 4.1). Igualmente conviene destacar la presencia de pintura a base de trazos gruesos verticales situados sobre todo en el gollete de las ollitas. Decoraciones de otro tipo son las que presentan principalmente los anafres y lebrillos a base de peine y formando bandas paralelas y ondas, junto con impresiones y ungulaciones en el borde.

En cuanto a la cerámica vidriada se refiere conviene destacar algún fragmento de "verde y manganeso", en concreto los aparecidos en el silo A-I. Abundan por otra parte las cerámicas decoradas con la técnica de "cuerda seca" ya sea total o parcial si bien hay que reseñar que abunda más ésta última. De éstas constatamos la existencia de un fragmento con decoración epigráfica. Varios fragmentos de cerámica estampillada, decoraciones vegetales a base de manganeso sobre vedrío melado en los ataifores (fig. 2.1), y goterones de vedrío en la mayoría de los candiles completan las decoraciones más destacables en cuanto a la cerámica vidriada se refiere.

Parte de las figuras que acompañan al texto corresponden a materiales un tanto representativos o innovadores y que, en cualquier caso, pasaremos a describir con mayor detalle.

En la figura 3.2 se representa un cuchillo de cocina fabricado sobre lámina de hierro forjada. La parte superior de la hoja es asimétrica presentando un sólo filo. Para la fabricación del mango se adosaron sendas piezas de hueso a un lado y otro de la zona inferior de la hoja, siendo remachadas con clavos de hierro. Las cachas de hueso están decoradas con círculos concéntricos. Se trata de la primera pieza completa de este tipo, ya que únicamente se habian localizado fragmentos, como el aparecido en la Alcazaba de Badajoz.

Un probable fragmento de morillo ilustra la figura 3.1. Presenta incisiones a base de líneas rectas y zig-zags. Su función consiste en levantar la leña del suelo para facilitar su aireación y mejorar la combustión de forma notable. La zona más próxima al fuego presentaba antes de su restauración una cocción reductora muy consistente en relación al aspecto más deleznable de la zona más alejada. Esto se podría explicar mediante una cocción post-uso; es decir, el barro en crudo pero seco sería puesto en funcionamiento y el calor aportado en sucesivas ocasiones iría endureciendo la pieza. La zona inferior entre las patas y de forma semi-circular presenta en su interior un aspecto patinado por lo que podría haber sido apoyado en algún tipo de soporte. 

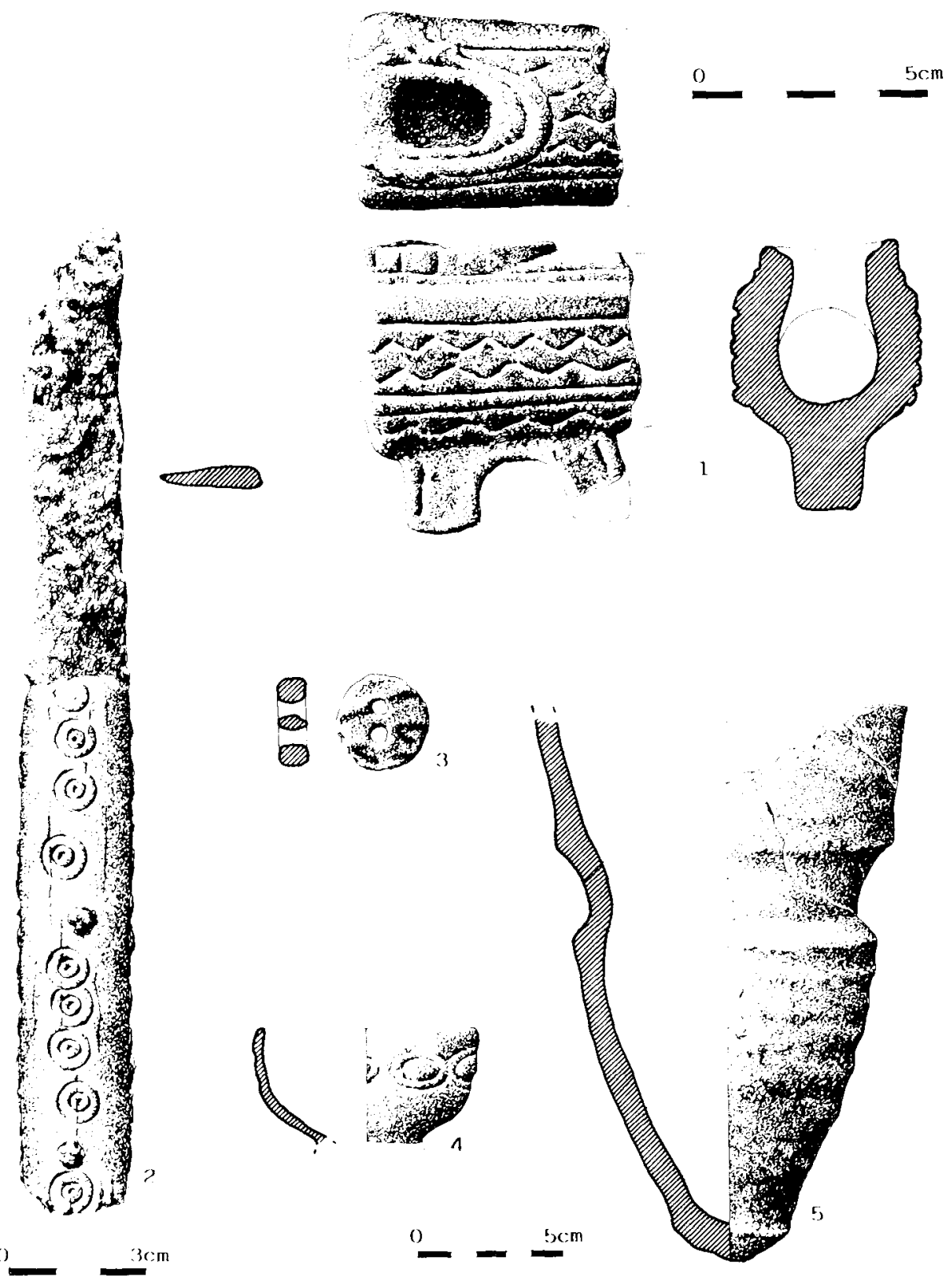

Fig. 3.1. Probable fragmento de morillo; 2. Cuchillo de hierro con cachas de hueso decoradas; 3. Botón de cerámica vidriada; 4. Cuenco de vidrio decorado; 5. Arcaduz o cangilón. Dibujo de Miguel Angel López Marcos. 

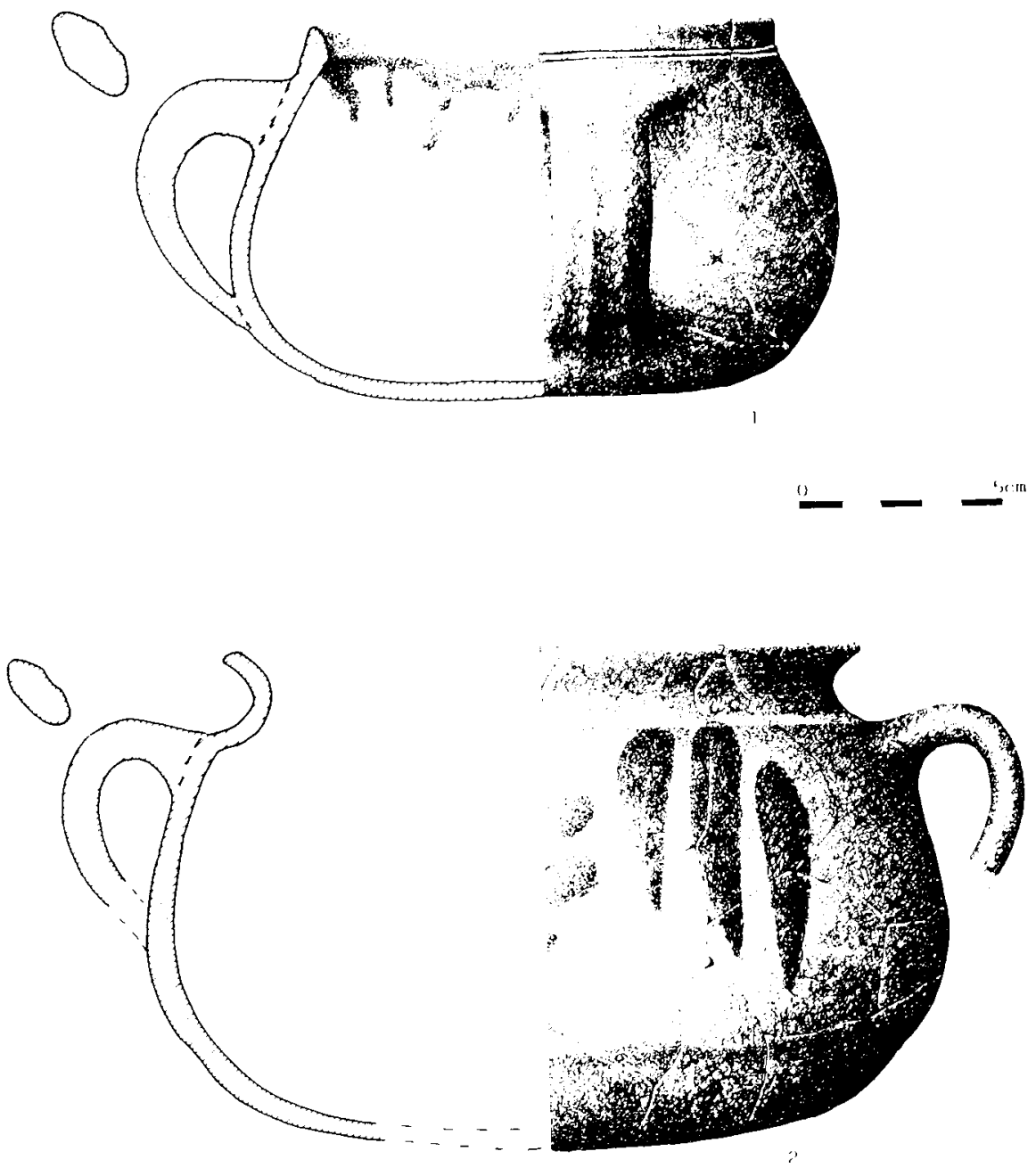

Fig. 4. Cerámica común o de cocina. Dibujo de Miguel Angel López Marcos. 

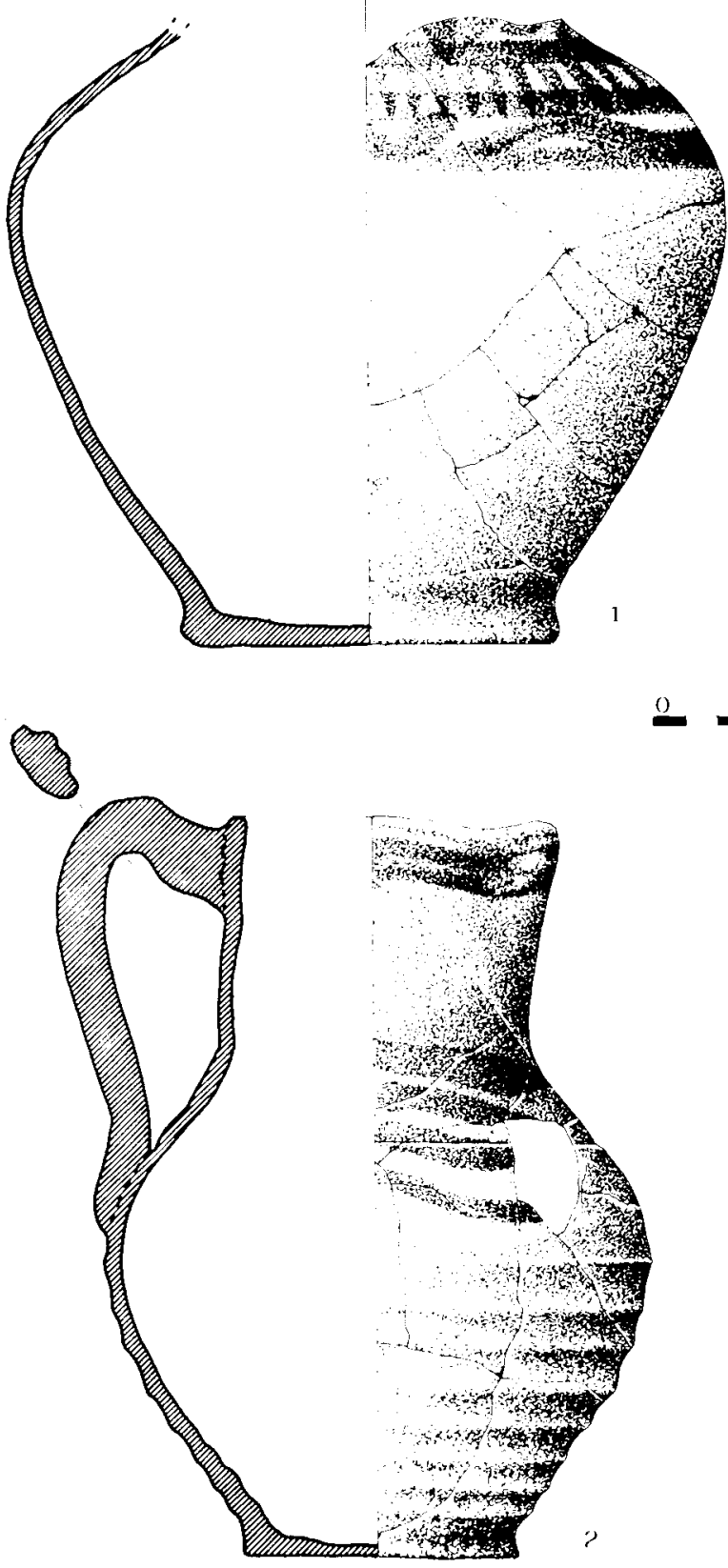

$0.5 \mathrm{sm}$

Fig. 5. Cerámica pintada de "repoblación". Dibujo de Miguel Angel López Mar$\cos$. 
El fragmento de vidrio de la figura 3.4 corresponde a un cuenco decorado, de pequeño tamaño y con un ensanchamiento inferior que indicaría el arranque de un repié o base engrosada. La decoración se compone de impresiones a base de pequeños ovalos concéntricos dispuestos en sentido longitudinal. Desde un punto de vista hialotécnico se trata de un vidrio soplado, recibiendo las impresiones con la "frita" aun caliente, por medio de las repetidas aplicaciones de un instrumento con la forma deseada. Presenta un aspecto verdoso claro debido a la adición de algún colorante como los óxidos de cobre, de cromo, de hierro o de niquel.

Cangilón o arcaduz para uso de noria, falta de la escotadura superior y el borde (fig. 3.5). Varios han aparecido de este tipo siendo el aquí representado el más completo. En la noria de la Huerta de Oliva (Valencia) encontramos un claro paralelo en cuanto a forma y tamaño, y está fechado entre los siglos $x$ y $x$ I. Tipos más cercanos encontramos en el testar de Toledo. Podría estar asociado a zonas de cultivo. 


\section{BIBLIOGRAFÍA}

Aguado, J.: La cerámica hispanomusulmana de Toledo. CSIC. Madrid, IPET, Toledo 1983.

Amador de los Rios y Rada Delgado, J.: Historia de la Villa y Corte de Madrid, Madrid 1860-1864.

Azcárate, J. M.: “San Andrés", Madrid, Madrid 1978.

BAzzANA, A.: "Una noria árabe en la Huerta de Oliva (Valencia)", Congreso de Arqueologia Medieval, pág. 421-432 Madrid 1987.

CABALlero, L. y otros: "Las murallas de Madrid. Excavaciones y estudios arqueológicos (1972-1982)", EPAM, Madrid 1983, pág. 43-113.

CABAllero, L. y TURINA, A.: "Informe previo de la prospección realizada en el solar de la $\mathrm{C} /$ Cava Baja 22 con vuelta a la $\mathrm{C} /$ Almendro 3 (Septiembre 1983)", EPAM, Madrid 1984, pág. 159-168.

Caballero, L. y otros: "Madrid: Barrio histórico. Informe de las excavaciones arqueológicas efectuadas en la plaza de los Carros (Nov-Dic 1983)", EPAM, Madrid, 1985, pág. 169-189.

IZQUIERDO, R.: "Los baños árabes de los Vascos (Navalmoralejo, Toledo)», NAH, núm. 28, Madrid 1987, pág. 193-231.

LLUVIA, L. M.: Cerámica medieval española. Barcelona 1973.

Martínez Alfaro, P. E.: "Historia del abastecimiento de aguas a Madrid.

El papel de las aguas subterráneas", AIEM, Madrid 1987.

MARTINEZ Lillo, S.: "Horno cerámico islámico número 1 del circo romano de Toledo", Madrid 1985.

MOLINA, L.: Una descripción anónima de Al-Andalus. Madrid 1983.

Moreno GarRido, M. J.: "La cerámica de cuerda seca peninsular, origen y dispersión", Congreso de Arqueología medieval, Madrid, 1987, pág. 33-42.

Oliver Asin, J.: Historia del nombre de Madrid. Madrid 1954.

Priego, C.: "Cerámica islámica madrileña en las antiguas colecciones del Museo Municipal", Congreso de Arqueologia Medieval, Madrid 1987, pág. 81-92. 
Pavón Maldonado, B.: "Miscelánea de arte y arqueologia musulmana", Al-Qantara 1980, pág. 46-61.

RETUERCE, $M$.: "Informe sobre la excavación arqueológica efectuada en el solar de la Cuesta de la Vega-Calle Mayor", Villa de Madrid, año XXIII, 1985.

- "La cerámica islámica de Calatalifa. Apunte sobre los Grupos cerámicos de la Marca Media", Bol. MAN, 1984, pág. 26-32.

- "Cerámicas islámicas procedentes de Torete (Guadalajara). Nuevos datos sobre los grupos cerámicos de la Marca Media", Bol. Asoc. Esp. de orientalistas XX, Burgos 1984.

Rosello Bordoy, G.: Ensayo de sistematización de la cerámica árabe de Mallorca. Palma de Mallorca 1978.

Rubio VISIERS, A.: "Cerámica de engalba blanca bajo cubierta", CAM, 1987.

TURinA, A.: "Cerámicas pintadas de Alcalá la Vieja, Alcalá de Henares", Congreso de Arqueología Medieval, Madrid 1987, pág. 753-762.

VALDÉS, F.: "La Alcazaba de Badajoz. Hallazgos islámicos", EAE, N. ${ }^{\circ}$ 144 1986, pág. 113-279.

Verdú, M.: "Algunas consideraciones en torno a los viajes de agua madrileños (1690-1750). Diseños de J. M. del Olmo y J. B. Sachetti para el arca principal del Bajo Abroñigal», AIEM, Madrid 1984, pág. 215-226.

ZOZAYA, J.: "Excavaciones en la fortaleza de Qal'at Abd-al-Salam (Alcalá de Henares, Madrid)», NAH, núm. 17, Madrid 1983, pág. 411-517. 